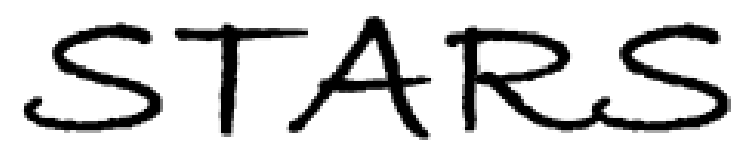

University of Central Florida

STARS

$1-1-2003$

\title{
Observation of multiple soliton generation mediated by amplification of asymmetries
}

\author{
Silvia Carrasco \\ University of Central Florida \\ Sergey Polyakov \\ University of Central Florida \\ Hongki Kim \\ University of Central Florida \\ Ladislav Jankovic \\ University of Central Florida \\ George I. Stegeman \\ University of Central Florida \\ Find similar works at: https://stars.library.ucf.edu/facultybib2000 \\ University of Central Florida Libraries http://library.ucf.edu \\ See next page for additional authors
}

This Article is brought to you for free and open access by the Faculty Bibliography at STARS. It has been accepted for inclusion in Faculty Bibliography 2000s by an authorized administrator of STARS. For more information, please contactSTARS@ucf.edu.

\section{Recommended Citation}

Carrasco, Silvia; Polyakov, Sergey; Kim, Hongki; Jankovic, Ladislav; Stegeman, George I.; Torres, Juan P.; Torner, Lluis; Katz, Mordechai; and Eger, David, "Observation of multiple soliton generation mediated by amplification of asymmetries" (2003). Faculty Bibliography 2000 s. 3657.

https://stars.library.ucf.edu/facultybib2000/3657

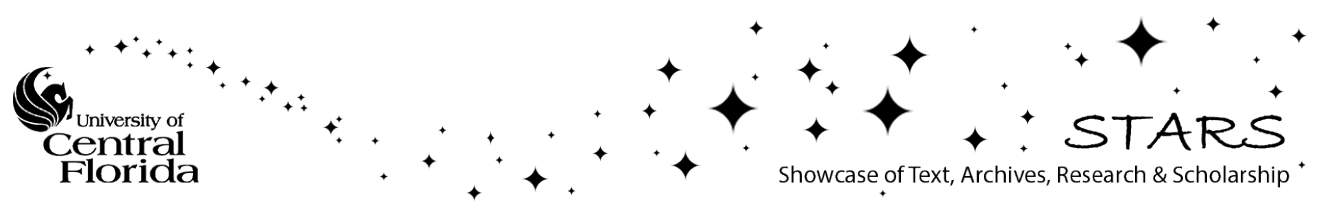




\section{Authors}

Silvia Carrasco, Sergey Polyakov, Hongki Kim, Ladislav Jankovic, George I. Stegeman, Juan P. Torres, Lluis Torner, Mordechai Katz, and David Eger 


\title{
Observation of multiple soliton generation mediated by amplification of asymmetries
}

\author{
Silvia Carrasco, Sergey Polyakov, Hongki Kim, Ladislav Jankovic, and George I. Stegeman \\ CREOL/School of Optics, University of Central Florida, Orlando, Florida 32816 \\ Juan P. Torres and Lluis Torner \\ ICFO-Institut de Ciencies Fotoniques, and Department of Signal Theory and Communications, Universitat Politecnica de Catalunya, \\ 08034 Barcelona, Spain \\ Mordechai Katz and David Eger \\ Electro-Optic Division, Soreq NRC, Yavene 81800, Israel \\ (Received 29 November 2002; published 25 April 2003)
}

\begin{abstract}
We report the experimental observation of the formation of multiple optical quadratic solitons in a process mediated by the amplification of minute asymmetries in the diffraction properties of the input light. Experiments were conducted in phase-matched second-harmonic generation in a bulk crystal of periodically poled potassium titanyl phosphate pumped at $1064 \mathrm{~nm}$. The different mechanisms that influence the process were investigated numerically, and the pulsed nature of the pump light was found to play a key role in the observed light distributions.
\end{abstract}

DOI: 10.1103/PhysRevE.67.046616

PACS number(s): 42.65.Tg, 42.65.Jx, 42.65.Wi

Solitons play a crucial role in many branches of nonlinear science $[1,2]$. They form by the proper balance between linear spreading effects and nonlinearity in a variety of physical systems. In this context, nonlinear optics and atomic physics occupy a very special position. First, because they provide a unique laboratory to experimentally explore the frontiers of the field. Second, because findings are important for a variety of applications, from future signal processing devices to the understanding of the dynamics of Bose-Einstein condensates [3-5], respectively. The first stage of the program is the identification of scenarios where robust solitons exist. A second crucial step is the elucidation of the dynamical routes that mediate the generation of solitons from accessible initial conditions. Two major routes abound in nature: On one hand, single solitons of Hamiltonian systems are generated by the reshaping, accompanied by radiation, of initial conditions into a soliton state, a process driven by the convergence towards the minimum of the field energy (see Refs. [1], [2], in general, and Ref. [6] for observations in the case of quadratic solitons addressed here). On the other hand, multiple soliton patterns can be generated by modulational instabilities, namely, the amplification of periodic modulations imprinted in constant initial conditions (see Ref. [7] for observations in the case of quadratic solitons). In this Letter we report the observation of a new route to the generation of multiple solitons, namely, the spontaneous amplification of small asymmetries of the diffraction properties of the beams.

Our experiments were conducted in periodically poled potassium titanyl phosphate (PPKTP). In this material and geometry, the relevant nonlinear coefficient is relatively large, thus input powers up to several times the soliton threshold can be achieved before the cubic nonlinearities and twophoton absorption (TPA) at the green wavelengths become significant. Single soliton formation in PPKTP has been recently observed under conditions of second-harmonic generation (SHG) [8], with peak intensity thresholds of about 3 $\mathrm{GW} / \mathrm{cm}^{2}$ with $18 \mu \mathrm{m}$ waist input beams. Single solitons emerge from Gaussian input beams, which reshape and radiate the extra energy away. With symmetrical beams the radiation is shed away in the form of rings that slowly diffract, thus leaving a single soliton in the output light pattern. However, our experimental investigations revealed that such is not necessarily the case in the presence of minute anisotropies in the diffraction properties of the beams induced, e.g., by a small astigmatism on the input beams. The interpretation of the experimental observations requires a detailed study of the light evolution inside the crystal, thus we first report the salient points of our numerical investigations. We modeled the light evolution in the crystal under the conditions of the experiments, namely, quasi-phase-matched SHG pumped with pulsed light in PPKTP, by solving the corresponding $(3+1)$-dimensional equations (we solved the equations given in Ref. [9] augmented with the temporal evolution and anisotropy; see Refs. [10-13] for further details). The small cubic nonlinearity and TPA of PPKTP were found to affect the concrete pattern of light, but did not modify the main features, thus all simulations shown here correspond to quadratic nonlinearities only. First we discuss the simulations in the case of $\mathrm{cw}$ pumping, and later the full spatiotemporal calculations. As in the experiments, we restrict ourselves to input conditions with very small ellipticities.

First, we study the case of a slightly asymmetric beam pumping a crystal with isotropic diffraction. The calculations show that within a range of input powers, several extra solitons are generated via two different ways. The most fascinating is the slow collapse into solitons of the radiation ring. Figure 1(a) shows an illustrative example. It corresponds to a Gaussian-like input beam with ellipticity $w_{y} / w_{x}=1.03$, where $w_{y}$ and $w_{x}$ are the beam widths. Notice that for an actual beam waist of $20 \mu \mathrm{m}$, such difference between $w_{y}$ and $w_{x}$ corresponds to only half a micrometer. The asymmetric radiation ring slowly evolves into two high-power spots that eventually form two solitons, besides the central spot. Thus, 
(a)
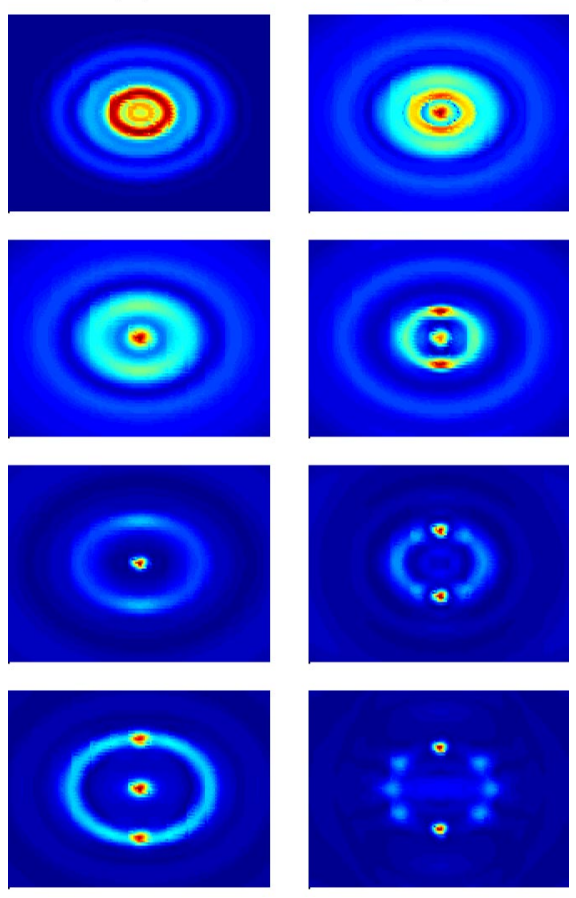

FIG. 1. CW simulations: SH profiles for different propagation distances inside the crystal. The FF input beam has a Gaussian shape with two different ellipticities: (a) $w_{y} / w_{x}=1.03$; (b) $w_{y} / w_{x}$ $=1.07$. The evolution is displayed at 3, 6, 9, and 12 diffraction lengths (from top to bottom). Conditions: up-conversion; exact phase matching; input field amplitude $a_{1}=22$. All definitions are as in Ref. [9].

the output light pattern typically has three solitons. Figure 1(b) shows a different route, where the input main peak breaks into several subbeams during the initial propagation units. Such subbeams undergo complex dynamics, which might include merging and splitting along the opposite axis, and eventually lead to the formation of several solitons. Along this route the generation of the central soliton is typically inhibited, thus leaving an output pattern with, e.g., two solitons. In the simulations, the system takes either of the routes depending on most of the parameters involved, including the values of the ellipticity and pump power. Thus, the output soliton pattern is also found to depend on these parameters. In any case, the transverse axis along which the soliton patterns are generated is dictated by the direction of the input beam asymmetry.

According to the above, the input asymmetries required to trigger the multiple soliton formation are very small. Thus, they might cooperate or compete with the anisotropic diffraction properties of the crystal, originated by the small difference in the refraction indexes along the propagation and the polarization directions [13]. A summary of the impact of this anisotropy on the light evolution in the presence of small input asymmetries is shown in Fig. 2. The plot corresponds to a fixed input $\mathrm{cw}$ power and shows the pattern of light predicted by the simulations at 10 diffraction lengths, for different strengths of the anisotropy and of the input beam asymmetries. For the particular value of the input power con-

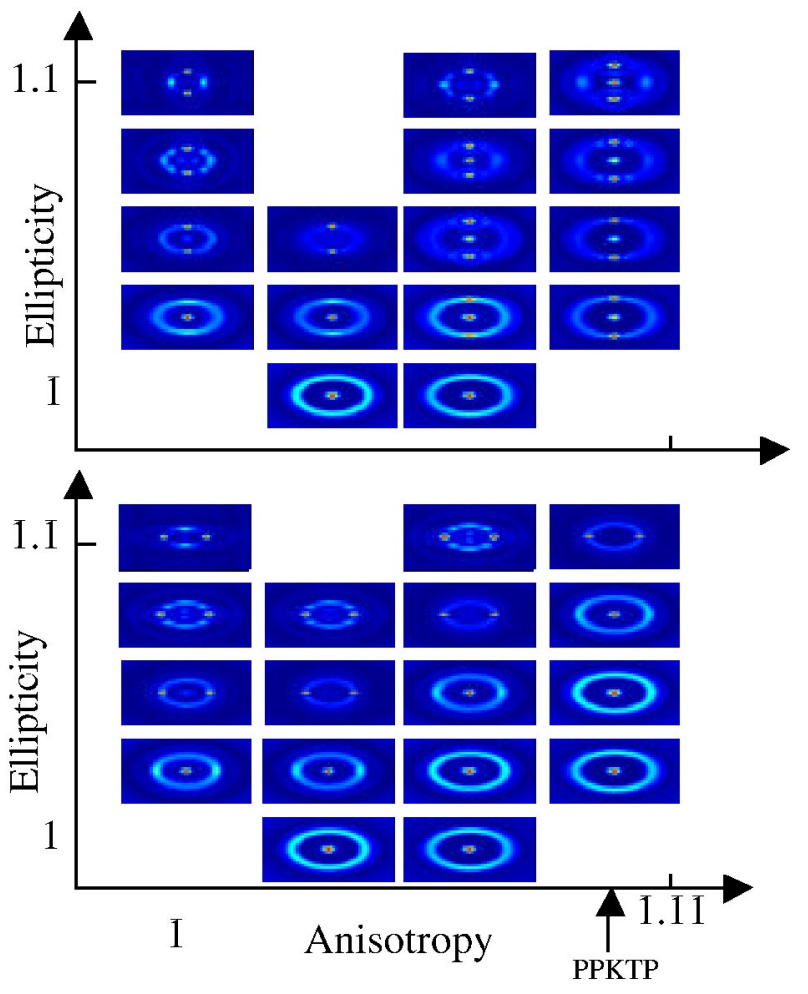

FIG. 2. CW simulations: SH profiles at 10 diffraction lengths for a fixed input power and several input beam asymmetries and material anisotropies. The horizontal axis is the normalized diffraction coefficient for the FF beam along the $x$ axis, $\alpha_{1 x}$. Additional diffraction coefficients: $\alpha_{1 y}=-1, \alpha_{2 x}=\alpha_{1 x} / 2, \alpha_{2 y}=-0.5$. All definitions are as in Ref. [13]. The vertical axis is the ratio $w_{y} / w_{x}$ in the upper figure and $w_{x} / w_{y}$ in the lower figure. Conditions are as in Fig. 1.

sidered, the plot shows that the anisotropy of PPKTP alone is not strong enough to generate multiple solitons at 10 diffraction lengths; only a radiation ring is visible. Solitons are predicted to form, via slow collapse of the ring, when one propagates further, e.g., until 20 diffraction lengths (a case that is not shown in the plot). The presence of small ellipticities in the input beam can speed up the process if the orientation of the ellipse coincides with the axis favored by crystal anisotropy. Then, anisotropy and input asymmetry cooperate. When the input ellipticities are set along the perpendicular axis both processes compete with each other, inhibiting the formation of additional solitons, or causing the additional solitons to be formed along the direction dictated solely by the asymmetry. Because the anisotropic diffraction of PPKTP is small, the latter is predicted to be a case for all realistic values of the input beam asymmetry. Thus, the input beam asymmetries are expected to be the dominant factor of the experiments, a result confirmed with our observations.

A key outcome of the numerical simulations is that the route taken towards multiple soliton formation (i.e., slow coalescence from the radiation ring or fast breakup of the main input peak) and thus the number of solitons present at the output light pattern, depends on the input power. Figure 3 illustrates the point. It corresponds to a geometry of cooperating input beam asymmetry and anisotropy, and shows the 
(a)
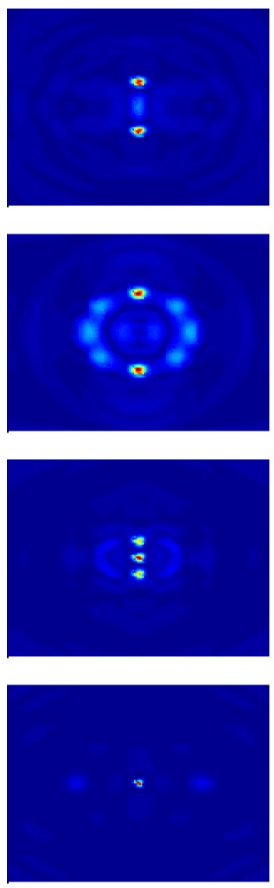

(b)
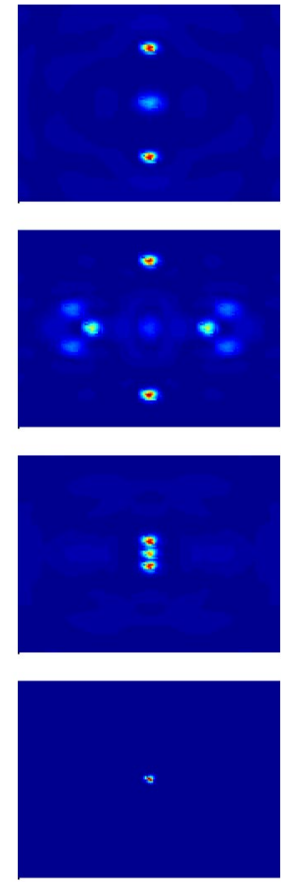

FIG. 3. CW simulations: SH profiles at (a) 10 and (b) 20 diffraction lengths, for four different input powers (normalized amplitudes $a_{1}=18,24,26,28$ from top to bottom) in an anisotropic medium with a small ellipticity present at the input, $w_{y} / w_{x}=1.03$. Conditions are as in Fig. 1. The anisotropic diffraction coefficients are those of PPKTP at 1064 and $532 \mathrm{~nm}: \alpha_{1 x}=-1.106, \alpha_{1 y}$ $=-1, \alpha_{2 x}=-0.5625, \alpha_{2 y}=-0.5$.

predicted light pattern for different input powers and two crystal lengths. The key question that arises is whether the above-mentioned routes might take place together in the actual case of pulsed pump light. To elucidate this point we conducted series of fully three-dimensional simulations with sech-shaped temporal pulses. We set a pulse duration of 25 ps thus a negligible group-velocity dispersion and groupvelocity mismatch. Figure 4 shows a typical outcome. It displays different spatial snapshots taken at selected temporal instances along the pulse, together with the plot obtained by temporal integration. The plot confirms that the different parts of the pulse undergo different dynamical routes. In the particular case shown, the two off-axis spots present in the temporally integrated image come from the fast branching into two spatial solitons of the pulse peak, while the central soliton comes from the pulse wings.

Our experimental observations were conducted in temperature-tuned, phase-matched $\mathrm{SHG}$ in a $1 \mathrm{~cm}$ long PPKTP crystal, fabricated using the low temperature periodic poling technique developed at Soreq and Tel-Aviv University to give a $d_{\mathrm{eff}}\left(=2 d_{33} / \pi\right)$ of $9.5 \mathrm{pm} / \mathrm{V}$ [14]. For a poling period of $\Lambda=9.0 \mu \mathrm{m}$, we measured that phase-matching occurred around $42.7^{\circ} \mathrm{C}$. An EKSMA Nd:YAG laser-amplifier delivering 25 ps pulses with a $10 \mathrm{~Hz}$ reprate was used to pump the crystal. The Z-polarized $1064 \mathrm{~nm}$ beam was focused to a beam waist (half-width at $1 / e^{2}$ in intensity) $w_{0}$
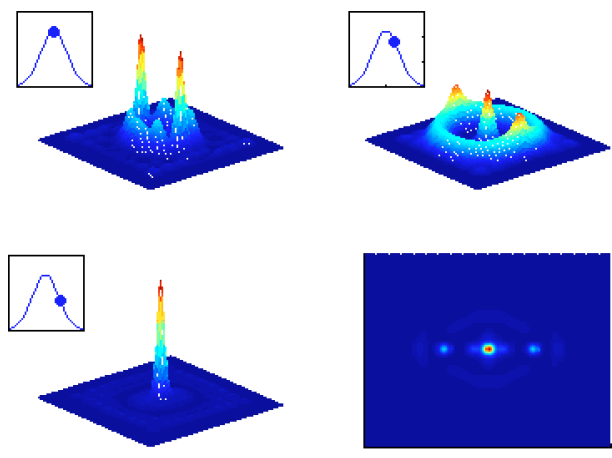

FIG. 4. Full three-dimensional, spatiotemporal simulations: spatial profile of the FF beam acquired at different temporal snapshots. The insets sketch the shape of the pulse, and the dots show the location of the temporal slice shown. The panel of the right-bottom corner is the spatial intensity profile calculated by temporal integration along the pulse. The input profile features a sech in time and a Gaussian in space. The ellipticity $w_{y} / w_{x}=1.07$. All other conditions are as in Fig. 1.

$=16.5 \mu \mathrm{m}$ at the input facet of the crystal, as measured by a knife-edge method, giving about 7.5 diffraction lengths inside the crystal for propagation along the $X$ axis. Figure 5 shows the central experimental observations. All pictures correspond to the same input intensity. With a very symmetric input beam (the best beam we could achieve in the lab) no additional solitons formed at the end of the crystal (upper row). However, when a very small asymmetry, barely distinguishable in the CCD (charge coupled device) images, was imposed to the beam by an asymmetric pinhole, additional solitons formed along anaxis (central row). That the multiple
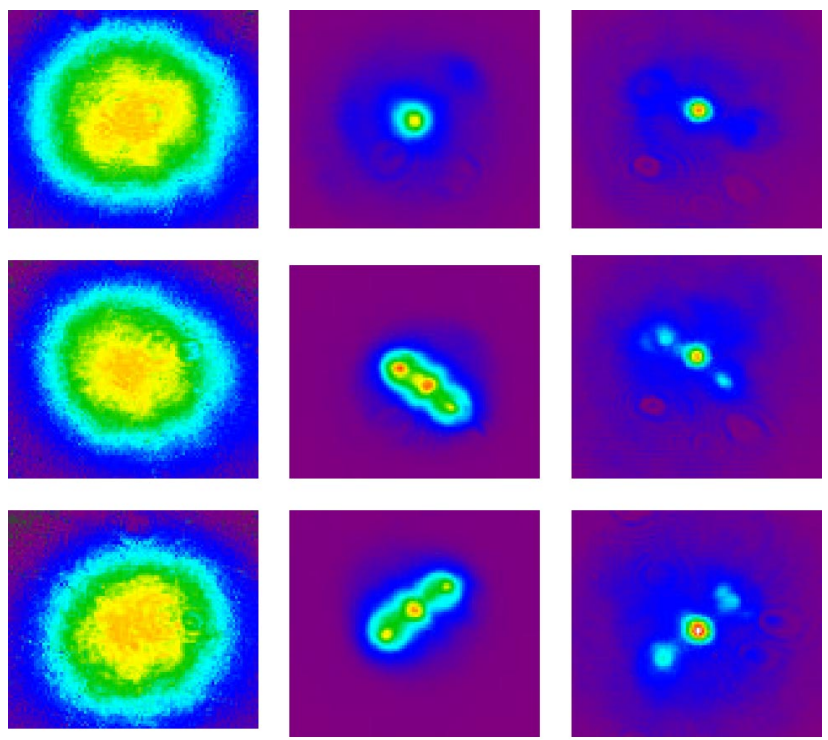

FIG. 5. Experiments: input FF (left), and output FF and SH (center and right, respectively). Upper row: very symmetric input beam; center, slightly input asymmetric; bottom, same as in center but rotated $90^{\circ}$. Input peak intensity, $23 \mathrm{GW} / \mathrm{cm}^{2}$; input beam waist, $16.5 \mu \mathrm{m}$; near phase matching. 
solitons arise from the amplification of the small asymmetry of the input beam was revealed by rotating the input beam $90^{\circ}$ with a Dove prism (lower row): Then the additional solitons were aligned along the rotated axis. Consistent with the numerical simulations, multiple solitons were observed only near exact phase matching, and inside a band of input intensities, which might vary with the crystal length (i.e., solitons might be still not formed at too short lengths), and with the beam ellipticity. Temporally resolved data acquired under related conditions [15] support the conclusion that the observed light pattern is mediated by a combination of spatiotemporal dynamics.
We conclude noticing that although our observations of the multiple soliton generation mediated by the amplification of diffraction asymmetries were conducted with light, the route uncovered is relevant to other similar systems, in particular, to atomic-molecular Bose-Einstein condensates [16]. The matter-wave analog of our observations would correspond to the formation of multiple drops of quantum condensed coherent atoms and molecules, self-sustained without a trap.

This research was supported by an ARO MURI grant in the U.S., by the Generalitat de Catalunya, and by the Spanish Government through Grant No. BFM2002-2861.
[1] M. J. Ablowitz and P. A. Clarkson, Solitons, Nonlinear Evolution Equations and Inverse Scattering (Cambridge University Press, Cambridge, UK, 1991).

[2] N. N. Akhmediev and A. Ankiewicz, Solitons (Chapman-Hall, London, 1997).

[3] M. Segev and G. I. Stegeman, Phys. Today 51(8), 42 (1998); see also Solitons, Opt. Photonics News 13(2), 27 (2002), special issue.

[4] R. A. Battye and P. M. Sutcliffe, Phys. Rev. Lett. 86, 3989 (2001)

[5] K. E. Strecker et al., Nature (London) 417, 150 (2002); L. Khaykovich et al., Science 296, 1290 (2002); J. R. Anglin and W. Ketterle, Nature (London) 416, 211 (2002); B. P. Anderson and P. Meystre, Opt. Photonics News 13, 20 (2002).

[6] W. E. Torruellas et al., Phys. Rev. Lett. 74, 5036 (1995); R. Schiek et al., Phys. Rev. E 53, 1138 (1996); R. A. Fuerst et al., Opt. Lett. 22, 1748 (1997); P. Di Trapani et al., Phys. Rev. Lett. 80, 265 (1998); X. Liu et al., ibid. 82, 4631 (1999); E. Lopez-Lago et al., Opt. Lett. 26, 1277 (2001).

[7] R. A. Fuerst et al., Phys. Rev. Lett. 78, 2756 (1997); D. V. Petrov et al., Opt. Lett. 23, 1444 (1998); H. Fang et al., ibid.
25, 1786 (2000); X. Liu et al., Phys. Rev. Lett. 85, 1871 (2000).

[8] H. Kim et al., CLEO 2002, Long Beach, CA, paper CWL2, 2002.

[9] S. Carrasco et al., Opt. Lett. 27, 2016 (2002).

[10] C. R. Menyuk et al., J. Opt. Soc. Am. B 11, 2434 (1994); O. Bang, ibid. 14, 51 (1997).

[11] H. Li et al., Opt. Commun. 144, 75 (1997).

[12] A. V. Buryak, P. Di Trapani, D. Skryabin, and S. Trillo, Phys. Rep. 370, 63 (2002).

[13] S. Polyakov et al., Opt. Lett. 27, 1049 (2002); Phys. Rev. E 66, 046622 (2002).

[14] G. Rosenman et al., Appl. Phys. Lett. 73, 3650 (1998).

[15] S. Minardi and P. Di Trapani (private communication).

[16] Observations which are claimed to be due to the occurrence of coherence in atomic-molecular Bose-Einstein condensates have been reported recently in E.A. Donley et al., Nature (London) 417, 529 (2002); see B. J. Cusack et al., Phys. Rev. A 65, 013609 (2002), and references therein for mathematical models. 